Article

\title{
A New Image Processing Procedure Integrating PCI-RPC and ArcGIS-Spline Tools to Improve the Orthorectification Accuracy of High-Resolution Satellite Imagery
}

\author{
Hongying Zhang ${ }^{1,2}$, Ruiliang $\mathrm{Pu}^{2}$ and Xiuguo Liu ${ }^{1, *}$ \\ 1 Faculty of Information Engineering, China University of Geosciences (Wuhan), No. 388 Lumo Road, \\ Wuhan 430074, China; yiwenxiaoshi@sina.com \\ 2 School of Geosciences, University of South Florida, 4202 East Fowler Avenue, NES107, Tampa, \\ FL 33620, USA; rpu@usf.edu \\ * Correspondence: liuxg318@hotmail.com; Tel.: +86-27-6788-3728; Fax: +86-27-6788-3809 \\ Academic Editors: Gonzalo Pajares Martinsanz, Xiaofeng Li and Prasad S. Thenkabail \\ Received: 21 June 2016; Accepted: 27 September 2016; Published: 9 October 2016
}

\begin{abstract}
Given the low accuracy of the traditional remote sensing image processing software when orthorectifying satellite images that cover mountainous areas, and in order to make a full use of mutually compatible and complementary characteristics of the remote sensing image processing software PCI-RPC (Rational Polynomial Coefficients) and ArcGIS-Spline, this study puts forward a new operational and effective image processing procedure to improve the accuracy of image orthorectification. The new procedure first processes raw image data into an orthorectified image using PCI with RPC model (PCI-RPC), and then the orthorectified image is further processed using ArcGIS with the Spline tool (ArcGIS-Spline). We used the high-resolution CBERS-02C satellite images (HR1 and HR2 scenes with a pixel size of $2 \mathrm{~m}$ ) acquired from Yangyuan County in Hebei Province of China to test the procedure. In this study, when separately using PCI-RPC and ArcGIS-Spline tools directly to process the HR1/HR2 raw images, the orthorectification accuracies (root mean square errors, RMSEs) for HR1/HR2 images were $2.94 \mathrm{~m} / 2.81 \mathrm{~m}$ and $4.65 \mathrm{~m} / 4.41 \mathrm{~m}$, respectively. However, when using our newly proposed procedure, the corresponding RMSEs could be reduced to $1.10 \mathrm{~m} / 1.07 \mathrm{~m}$. The experimental results demonstrated that the new image processing procedure which integrates PCI-RPC and ArcGIS-Spline tools could significantly improve image orthorectification accuracy. Therefore, in terms of practice, the new procedure has the potential to use existing software products to easily improve image orthorectification accuracy.
\end{abstract}

Keywords: high-resolution satellite imagery; orthorectification; PCI-RPC; ArcGIS-Spline

\section{Introduction}

With the development of remote sensing technology, the spatial resolution and spectral resolution of remote sensing images have been greatly improved, and thus application fields of remote sensing technology are expanding [1-3]. High resolution satellite remote sensing imagery is a basic spatial data source to construct the digital Earth and can be widely applied in multiple subjects and areas, such as geology, vegetation, agriculture, forestry, and oceanography, etc. [4-6], especially in disaster emergency monitoring, real-time monitoring of land cover, ocean monitoring, and Earth-crust displacement and ground settlement monitoring $[1,2,7,8]$.

Given the fact that high resolution satellite images possess the characteristics of timeliness and authenticity, rapidly acquiring information, relatively low cost, no geographic restrictions, and abundant spatial information and texture information, etc., developing high resolution satellite 
image processing techniques and conducting various application studies have continuously received attention [7-9]. However, it is still challenging for us to develop an effective and accurate image processing method in order to rapidly, automatically identify and extract useful information for various application purposes from processed remote sensing images $[7,10]$. In the processing of high resolution satellite images, investigating orthorectification models of satellite imaging is not only their core content but also a basis for high accurate orthorectification of remote sensing images [11]. This is because only if a mathematical model of satellite imaging is established can the mathematical relationship between three-dimensional spatial coordinates of ground control points (GCPs) and corresponding pixel coordinates of image points be reflected [12,13].

The mathematical models of satellite imaging are generally divided into two categories. One is a strict imaging geometry model based on imaging properties of a sensor, commonly referred to as a physical model, which is established according to a strict geometrical relationship among GCPs of imaging, the center of the sensor lens and the corresponding image points in a straight line [2]. The other is the general imaging geometry model only based on a simple mathematical function unrelated to the specific sensor, commonly referred to as a rational function model, which is established according to a relationship between GCPs and the corresponding image points [14,15]. Similar to the general imaging geometry model or called a rational function model, the homography transformation is also one of the most popular and efficient geometric transformation models, and is frequently used in alignment of images and related fields in a single coordinate system to acquire 3D information, to detect/measure geometric difference, or to increase the field of view or signal-to-noise ratio [16]. Typically, homographies are estimated between images by finding feature correspondences in those images. Homographic transformation of an image can be implemented by multiplying the image coordinate with the homography transform matrix [17]. Since the physical model is applicable for a collinear equation model, satellite orbit ephemeris parameters and sensor parameters are necessary. However, it is either very difficult or impossible for most users to obtain such parameters [18]. Therefore, nowadays rational function models are adopted by most users for an orthorectification of remote sensing images, and commonly used models include the polynomial correction model and the rational polynomial coefficients (RPC) correction model [2,15].

The polynomial correction model is more suitable for a plain area with a relatively smooth terrain because it does not need to consider the spatial geometry relation of imaging process. Rather, the RPC correction model is widely used in mountainous regions with a greater topographic relief due to the relief factor. Furthermore, the rectification accuracy of the RPC model is close to the collinear equation model [15,19]. However, there are some limitations for the RPC model. First, the RPC correction model is in the form of a fraction, so it may fail to work when the denominator is equal to zero [13,20]. Second, the RPC correction model may only correct errors of GCP but cannot eliminate the image distortion, so the accuracy of corrected images would be still affected by the accuracy of DEM [15]. Third, because the RPC correction model is established based on specific points, theoretically, it is not strictly applicable to other points [19]. Additionally, since the accuracy of the RPC model is dependent upon the GCP accuracy, distribution and quantity, it is necessary for users to have a set of high-quality GCPs running the PRC model $[13,18]$. Therefore, at present, for a high resolution satellite image covering a mountainous area with a greater relief, it is essential for users to increase the image orthorectification accuracy.

In this study, to improve the orthorectification result for high resolution satellite images covering mountainous areas, a new operational and accurate processing procedure was proposed and tested, and the proposed procedure made a full use of mutual complementary satellite image processing software functions of PCI-RPC and ArcGIS-Spline [21,22]. To do so, the raw image data were firstly processed into an orthorectified image using PCI with a RPC correction model, and then the orthorectified image was further processed using an ArcGIS-Spline tool for a local geometric correction [23,24]. Local geometric correction is one of the key technologies to improve the accuracy of image orthorectification. A correlation coefficient method is frequently adopted in the search area to obtain the most relevant 
points (i.e., GCPs) because of the good consistency and pixel-level accuracy of the orthorectified image. Therefore, the spline function is established for these points respectively to calculate the correct value and obtain local geometric corrected coordinates point-by-point, so as to improve the accuracy of image orthorectification [19]. Essentially, local geometric correction is used to transform physical coordinates to user coordinates so that the spatial information of ArcGIS database has a practical significance [2,25]. Finally, based on the tested results, the proposed procedure was evaluated and relevant issues were discussed.

\section{Methodology}

\subsection{Study Area}

The experimental area is located in Yangyuan County $\left(39^{\circ} 53^{\prime} \mathrm{N}-40^{\circ} 22^{\prime} \mathrm{N}, 113^{\circ} 54^{\prime} \mathrm{E}-114^{\circ} 48^{\prime} \mathrm{E}\right)$, which is in the transition zone of Loess Plateau, Inner Mongolia Plateau and the North China Plain, Figure 1a shows the location of the experimental region [7]. It is mountainous in the northern and southern portions of the county and Sanggan River runs across the whole county from west to east. The geomorphic types include mountains, hills, plains, and rivers, etc. The terrain of the experimental area is high in southwest, low in northeast, high in southern mountains and low in northern mountains. Because of the special topographical characteristics, it is difficult to get high-accuracy orthorectification results for high resolution satellite images covering the study area [18].

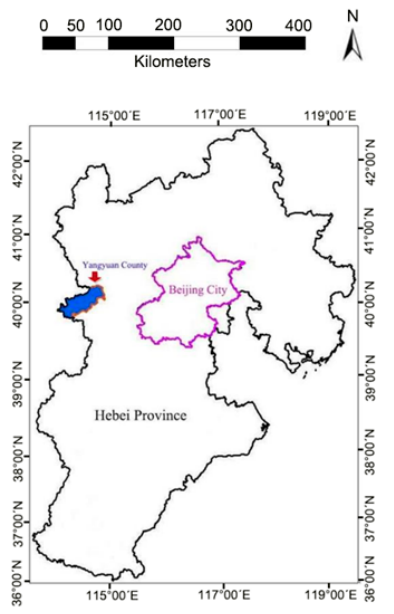

(a)

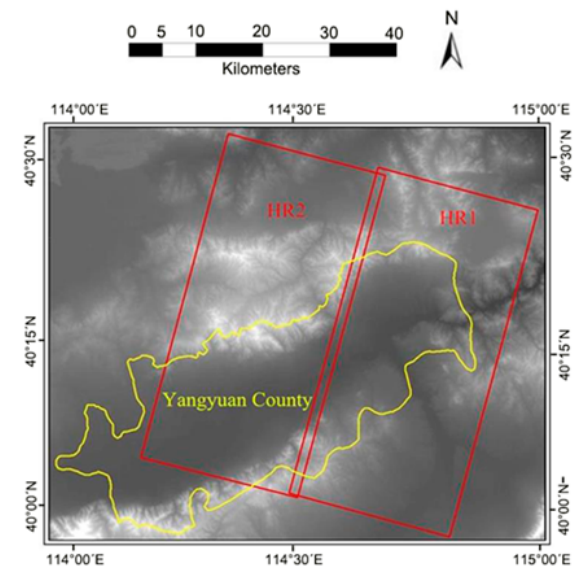

(c)

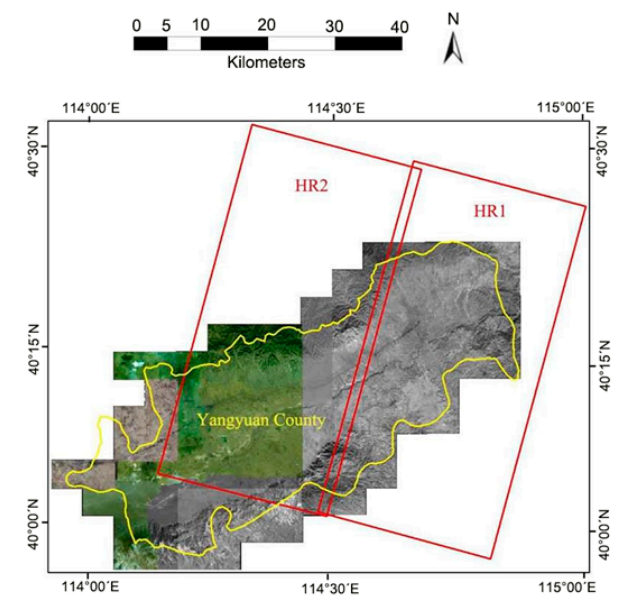

(b)

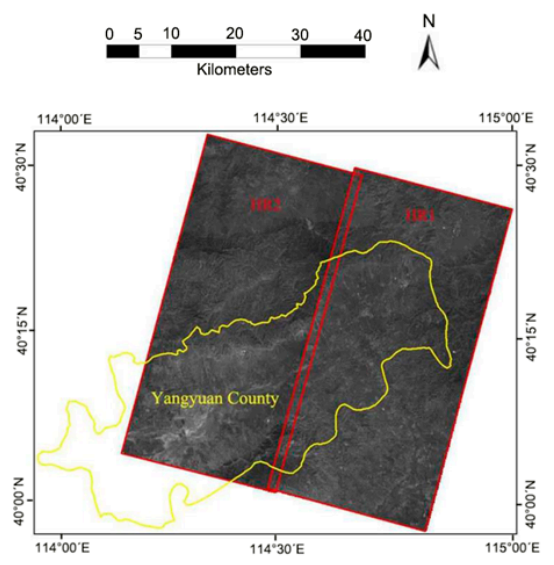

(d)

Figure 1. The location of the experimental area and presentation of experimentally used image data: (a) the location of the experimental area; (b) base image; (c) DEM data; and (d) warp images: HR1/HR2. 


\subsection{Data Sets}

In this study, the experimental data, including base image, high resolution warp image, and DEM (Digital Elevation Models) data, were collected and used as follows.

- $\quad$ Base image: An image of the second national land survey of China was collected to be used as a base image (Ministry of Land and Resources of People's Republic of China), which was shown in Figure $1 b$.

- Warp images: High resolution CBERS-02C HR satellite images, track number No. ZY02C-HRC-E114.6_N40.3_20140904_L1C0001836234, including two scenes, HR1 and HR2, with GeoTiff format were acquired from Yangyuan County, Hebei Province, China, on 4 September 2014, which was shown in Figure 1d. Both HR1 and HR2 scenes covered most of mountainous areas with a greater topographic relief. As China's first satellite of land and resources, CBERS-02C carries a P/MS multi-spectral camera and two high resolution cameras (HRCs). The P/MS cameras can provide multispectral data with $10 \mathrm{~m}$ spatial resolution (MUS image) covering visible and near-infrared spectral regions with three bands: band2 $(0.52-0.59 \mu \mathrm{m})$, band3 $(0.63-0.69 \mu \mathrm{m})$, and band $4(0.77-0.89 \mu \mathrm{m})$, and one panchromatic band image (PAN image) with $5 \mathrm{~m}$ spatial resolution covering spectral range $0.51-0.85 \mu \mathrm{m}$ (band1). The HRC cameras can acquire $2.36 \mathrm{~m}$ spatial resolution image, covering spectral range $0.50-0.80 \mu \mathrm{m}$ [26]. In this study, only two scenes of HRC images (HR1 and HR2) were tested.

- DEM data: DEM SRTM data with $30 \mathrm{~m}$ spatial resolution were collected, which are shown in Figure 1c [18]. So far, the DEM data are the best (resolution) we could use in the study area.

\subsection{Rectification Models}

Because the rational function models can run without satellite orbit and sensor parameters, they are widely used for orthorectification. Polynomial and RPC models are typical rational function models.

\subsubsection{Polynomial Rectification Model}

Polynomial rectification model is a mathematical model based on an image without regard to spatial geometry relationship of imaging process [27]. The quadratic polynomial model is frequently adopted in the modeling, which is established based on a relationship between ground coordinates $X$, $Y$ and image coordinates of control points $x, y$, as follows [14,28]:

$$
\left\{\begin{array}{l}
x=a_{00}+a_{10} X+a_{01} Y+a_{20} X^{2}+a_{11} X Y+a_{02} Y^{2} \\
y=b_{00}+b_{10} X+b_{01} Y+b_{20} X^{2}+b_{11} X Y+b_{02} Y^{2}
\end{array}\right.
$$

Based on the principle of least squares, the coefficients of quadratic polynomial $a_{00}, a_{10}, a_{01}, a_{20}$, $a_{11}, a_{02}, b_{00}, b_{10}, b_{01}, b_{20}, b_{11}, b_{02}$ can be solved by the Equation (1). Then the new coordinates of images can be calculated according to the transformation coefficients [14]. This method is usually adopted for plains with relatively smooth terrain, and is not recommended for mountainous regions. Therefore, it is not used for this study as this experimental area has a greater topographical relief [18].

\subsubsection{RPC Rectification Model}

(1) Expression of RPC Model

The RPC (Rational Polynomial Coefficients) model is based on ground control points (GCPs) and DEM data to orthorectify an image [14]. Actually, it is a broader and better expression of the sensor model, which is utilized to obtain the rows and lines of an image by the ratio and similar ratio of two polynomial functions respectively. Furthermore, both polynomials are the functions of ground coordinates. Therefore, the RPC model is for further generalization for polynomial model and linear transform model and it is suitable for different types of sensors [8,12]. In the RPC rectification model, 
the image coordinates are the ratio of two polynomials in which the three-dimensional coordinates of GCPs are set as independent variables as follows in Equation (2) [2,15,20,25,27,29,30]:

$$
\left\{\begin{array}{l}
r_{n}=\frac{P_{1}\left(X_{n}, Y_{n}, Z_{n}\right)}{P_{2}\left(X_{n}, Y_{n}, Z_{n}\right)} \\
c_{n}=\frac{P_{3}\left(X_{n}, Y_{n}, Z_{n}\right)}{P_{4}\left(X_{n}, Y_{n}, Z_{n}\right)}
\end{array}\right.
$$

where $\left(r_{n}, c_{n}\right)$ and $\left(X_{n}, Y_{n}, Z_{n}\right)$ are the normalized image coordinates $(r, c)$ and ground coordinates $(X, Y, Z)$ by translating and scaling in a RPC model $[20,29,31]$. Generally, ground coordinates and image coordinates are translated and scaled into parameter values between $(-1,1)$ to enhance the stability of parameter solutions, to reduce the computational errors caused by a large data magnitude, and to avoid causing a morbid matrix. The conversion relationship is shown in Equation $(3)[19,20,29]$ :

$$
\left\{\begin{aligned}
X_{n} & =\frac{X-X_{0}}{X_{s}} \\
Y_{n} & =\frac{Y-Y_{0}}{Y_{s}} \\
Z_{n} & =\frac{Z-Z_{0}}{Z_{s}} \\
r_{n} & =\frac{r-r_{0}}{r_{s}} \\
c_{n} & =\frac{c-c_{0}}{c_{s}}
\end{aligned}\right.
$$

where $\left(X_{0}, Y_{0}, Z_{0}, r_{0}, c_{0}\right)$ are the translating parameters of standardization, and also are the coordinates of the origin of a RPC model in the mapping coordinate system; $\left(X_{s}, Y_{s}, Z_{s}, r_{s}, c_{s}\right)$ are the proportional parameters of standardization. In the polynomial $P_{i}(X, Y, Z)(i=1,2,3,4)$, the maximum and the sum power of each coordinate component would not be greater than three [29]. According to Boccardo et al. [2], Hu et al. [15], Tao et al. [20], Li et al. [29], and Aguilar et al. [30], the polynomial representation is shown as follows:

$$
\begin{aligned}
P(X, Y, Z)= & a_{0}+a_{1} X+a_{2} Y+a_{3} Z+a_{4} X Y+a_{5} X Z+a_{6} Y Z+a_{7} X^{2}+a_{8} Y^{2}+a_{9} Z^{2}+a_{10} X Y Z+a_{11} X^{2} Y \\
& +a_{12} X^{2} Z+a_{13} Y^{2} X+a_{14} Y^{2} Z+a_{15} X Z^{2}+a_{16} Y Z^{2}+a_{17} X^{3}+a_{18} Y^{3}+a_{19} Z^{3}
\end{aligned}
$$

where the polynomial coefficients $a_{0}, a_{1}, \ldots, a_{19}$ are designated as the coefficients of the rational polynomial function.

(2) Othorectification Principle of the RPC Model

Substitute Equation (4) into Equation (3) and let $X_{n}=f_{x}(X)=\frac{X-X_{0}}{X_{S}}, Z_{n}=f_{z}(Z)=\frac{Z-Z_{0}}{Z_{S}}$, $Y_{n}=f_{y}(Y)=\frac{Y-Y_{0}}{Y_{S}}$, then $\frac{d X_{n}}{d X}=\frac{1}{X s}, \frac{d Y_{n}}{d Y}=\frac{1}{Y_{s}}, \frac{d Z_{n}}{d Z}=\frac{1}{Z s}$; substitute $r_{n}=\frac{r-r_{0}}{r_{s}}$ and $c_{n}=\frac{c-c_{0}}{c_{s}}$ into Equation (2), which can be written as:

$$
\left\{\begin{array}{l}
r=r_{s} \frac{P_{1}\left(X_{n}, Y_{n}, Z_{n}\right)}{P_{2}\left(X_{n}, Y_{n}, Z_{n}\right)}+r_{0} \\
c=c_{s} \frac{P_{3}\left(X_{n}, Y_{n}, Z_{n}\right)}{P_{4}\left(X_{n}, Y_{n}, Z_{n}\right)}+c_{0}
\end{array}\right.
$$

Let $F\left(X_{n}, Y_{n}, Z_{n}\right)=\frac{P_{1}\left(X_{n}, Y_{n}, Z_{n}\right)}{P_{2}\left(X_{n}, Y_{n}, Z_{n}\right)}$, and $G\left(X_{n}, Y_{n}, Z_{n}\right)=\frac{P_{3}\left(X_{n}, Y_{n}, Z_{n}\right)}{P_{4}\left(X_{n}, Y_{n}, Z_{n}\right)}$, then Equation (5) can be re-written as:

$$
\left\{\begin{array}{l}
r=r_{s} F\left(X_{n}, Y_{n}, Z_{n}\right)+r_{0} \\
c=c_{s} G\left(X_{n}, Y_{n}, Z_{n}\right)+c_{0}
\end{array}\right.
$$

Equation (6) may be written using theTaylorformula as follows:

$$
\left\{\begin{array}{l}
r=\hat{r}+\frac{\partial_{r}}{\partial_{X}} \Delta X+\frac{\partial_{r}}{\partial_{Y}} \Delta Y+\frac{\partial_{r}}{\partial_{Z}} \Delta Z+r_{0} \\
c=\hat{c}+\frac{\partial_{c}}{\partial_{X}} \Delta X+\frac{\partial_{c}}{\partial_{Y}} \Delta Y+\frac{\partial_{c}}{\partial_{Z}} \Delta Z+c_{0}
\end{array}\right.
$$


The error equation of Equation (7) is written as:

$$
\left\{\begin{array}{c}
v_{r}=\left[\begin{array}{lll}
\frac{\partial_{r}}{\partial_{X}} & \frac{\partial_{r}}{\partial_{Y}} & \frac{\partial_{r}}{\partial_{Z}}
\end{array}\right]\left[\begin{array}{c}
\Delta X \\
\Delta Y \\
\Delta Z
\end{array}\right]+(r-\hat{r}) \\
v_{c}=\left[\begin{array}{lll}
\frac{\partial_{c}}{\partial_{X}} & \frac{\partial_{c}}{\partial_{Y}} & \frac{\partial_{c}}{\partial_{Z}}
\end{array}\right]\left[\begin{array}{c}
\Delta X \\
\Delta Y \\
\Delta Z
\end{array}\right]+(c-\hat{c})
\end{array}\right.
$$

Equation (8) can be re-written as:

$$
\left[\begin{array}{c}
v_{r} \\
v_{c}
\end{array}\right]=\left[\begin{array}{lll}
\frac{\partial_{r}}{\partial_{X}} & \frac{\partial_{r}}{\partial Y} & \frac{\partial_{r}}{\partial_{Z}} \\
\frac{\partial_{c}}{\partial_{X}} & \frac{\partial_{c}}{\partial_{Y}} & \frac{\partial_{c}}{\partial_{Z}}
\end{array}\right]\left[\begin{array}{c}
\Delta X \\
\Delta Y \\
\Delta Z
\end{array}\right]+\left[\begin{array}{c}
r-\hat{r} \\
c-\hat{c}
\end{array}\right]
$$

And let $\boldsymbol{V}=\left[\begin{array}{c}v_{r} \\ v_{c}\end{array}\right], \boldsymbol{A}=\left[\begin{array}{ccc}\frac{\partial_{r}}{\partial_{X}} & \frac{\partial_{r}}{\partial_{Y}} & \frac{\partial_{r}}{\partial_{Z}} \\ \frac{\partial_{c}}{\partial_{X}} & \frac{\partial_{c}}{\partial_{Y}} & \frac{\partial_{c}}{\partial_{Z}}\end{array}\right], \Delta=\left[\begin{array}{c}\Delta X \\ \Delta Y \\ \Delta Z\end{array}\right], l=\left[\begin{array}{c}(r-\hat{r}) \\ (c-\hat{c})\end{array}\right]$, so Equation (9) finally can be written in a vector and matrix form as:

$$
V=A \Delta+l
$$

Based on Tao et al. [20] and Fraser et al. [13], the least squares solutions of coordinate corrections can be obtained from Equation (10) as follows:

$$
\Delta=\left[\begin{array}{lll}
\Delta X & \Delta Y & \Delta Z
\end{array}\right]^{T}=A^{-1}(V-l)=\left(A^{T} A\right)^{-1} A^{T}(V-l)
$$

Substituting Equation (11) into Equation (7), the coordinates of orthorectified image can be obtained. Because Equation (7) is a linearized model, iteration is operated to obtain an optimal solution for the image coordinates.

All above is the basic principle of the orthorectification model based on the RPC model, in which the projection distortion caused by the topographical relief can be rectified by combining the RPC model with DEM data [15]. Due to considering the relief factor, the rectification accuracy of the RPC model is only lower than that of a collinear equation model [13]. Given the experimental area covering more mountainous area with a greater topographical relief, this study adopted the RPC model to orthorectify both HR1 and HR2 images [13,20]. Although the RPC model can produce a higher accuracy of image orthorectification than the other models, due to the properties of the model itself, the PRC model still has some limitations, addressed in the introduction section.

\subsubsection{Spline Function Model}

A spline function is selected for local geometric correction because it can overcome the trouble of unstable and slow speed from high order interpolation [32]. The properties of minimum modulus and best approximation of the spline function can explain the resolution of the variational problem. Geometrically, the spline function describes a smooth-curved "thin beam" which "clamps" both end points through each interpolation point [33]. The cubic spline function is widely used because of its simple calculation, good stability, high precision, and certain smoothness.

Cubic spline function has two types of expression, first derivative and second derivative, both of which are continuous and have a unique solution. Suppose the spline function $S_{3, i}(x)$ is in a small interval $\left(x_{i-1}, x_{i}\right)(i=1,2, \ldots, n)$, which can be obtained by an interpolation condition as follows [34-37]: 


$$
\left\{\begin{array}{l}
S_{3, i}\left(x_{i-1}\right)=y_{i-1} \\
S_{3, i}\left(x_{i}\right)=y_{i}
\end{array} \quad(i=1,2, \ldots, n)\right.
$$

Let

$$
\left\{\begin{array}{l}
S^{\prime}{ }_{3, i}\left(x_{i-1}\right)=y^{\prime}{ }_{i-1}=m_{i-1} \\
S^{\prime}{ }_{3, i}\left(x_{i}\right)=y_{i}^{\prime}=m_{i}
\end{array} \quad(i=1,2, \ldots, n)\right.
$$

Then Equation (12) can be re-written by the Hermite's interpolation formula [34-36] as follows:

$$
\begin{gathered}
S_{3, i}(x)=m_{i-1} \frac{\left(x_{i}-x\right)^{2}\left(x-x_{i-1}\right)}{h_{i}^{2}}-m_{i} \frac{\left(x-x_{i-1}\right)^{2}\left(x_{i}-x\right)}{h_{i}^{2}}+y_{i-1} \frac{\left(x_{i}-x\right)^{2}\left[2\left(x-x_{i-1}\right)+h_{i}\right]}{h_{i}^{3}} \\
+y_{i} \frac{\left(x-x_{i-1}\right)^{2}\left[-2\left(x-x_{i}\right)+h_{i}\right]}{h_{i}^{3}}(i=1,2, \ldots, n)
\end{gathered}
$$

where $h_{i}=\left(x_{i}-x_{i-1}\right), x_{i-1} \leq x \leq x_{i}$. According to the definition of the cubic spline function, the second derivative is continuous, so it can be written as:

$$
\left\{\begin{array}{l}
S^{\prime \prime}{ }_{3}\left(x_{i-\sigma}\right)=S^{\prime \prime}{ }_{3}\left(x_{i+\sigma}\right) \\
S^{\prime \prime}{ }_{3 i}\left(x_{i}\right)=S^{\prime \prime}{ }_{3 i+1}\left(x_{i}\right)
\end{array}\right.
$$

Calculate the differential coefficient of Equation (14) and then substitute it into Equation (15) to obtain Equation (18) as follows:

$$
\lambda_{i} m_{i-1}+2 m_{i}+\mu_{i} m_{i+1}=C_{i}(i=1,2, \ldots, n)
$$

where $\mu=\frac{h_{i}}{h_{i}+h_{i+1}} ; \lambda_{i}=1-\mu_{i}$; and $C_{i}=3\left[\frac{\lambda_{i}\left(y_{i}-y_{i-1}\right)}{h_{i}}+\frac{\mu_{i}\left(y_{i+1}-y_{i}\right)}{h_{i+1}}\right],(1 \leq i \leq n-1)$.

Equation (18) contains $n-1$ equations with $n+1$ unknown variables, so it is necessary to add two more equations to obtain aunique solution, i.e., two boundary conditions. This model may adopt the first boundary condition, which is [37]:

$$
\left\{\begin{array}{l}
S_{3}^{\prime}\left(x_{0}\right)=y_{0}^{\prime}=m_{0} \\
S_{3}^{\prime}\left(x_{n}\right)=y_{n}^{\prime}=m_{n}
\end{array}\right.
$$

In this case, Equation (16) reduces two sum variables so that the unique solution for the equation can be obtained, and then the solution values of $m_{i}(i=1,2, \ldots, n)$ can be substituted into Equation (14) to establish a primary spline function.

In the procedure of orthorectification, the spline function relationship of $x, y$ can be established by Equation (14) according to the coordinates of the same GCPs identified from both base image and warp image [33,37]. Actually, the values of $m_{0}, m_{n+1}$ can be replaced by the reciprocal values of the slopes of the straight lines connected by the first two points and the last two points, respectively, and the values of $h_{i}$ depend on the situation. Finally, the correction values of $x, y$ can be calculated to correct coordinates of each GCP in a local area of an image [33].

\subsection{A Procedureof Orthorectification}

Figure 2 presents a flowchart to show the procedure of conducting orthorectification of images in this study $[28,38,39]$. In this study, firstly, we set the base image as a reference image, the CBERS-02C HR images as warp images and overlaid DEM data over both base and warp images. Secondly, RPC rectification model was adopted and satisfied GCPs were identified and collected from the both base image and warp images. Thirdly, the conversion model was calculated and the warp images were re-sampled. Finally, the warp images were orthorectified and outputted with an acceptable accuracy [40,41]. To do so, commercial software products PCI with RPC (Rational Polynomial Coefficients) model (PCI-RPC) and ArcGIS with Spline model (ArcGIS-Spline) were adopted to perform the proposed procedure of orthorectification of images. In order to improve 
the accuracy of orthorectifying satellite images that cover mountainous areas, we proposed a new operational and accurate processing procedure by making full use of the mutually compatible and complementary characteristics of the remote sensing image preprocessing software products PCI-RPC and ArcGIS-Spline tools $[23,24]$.

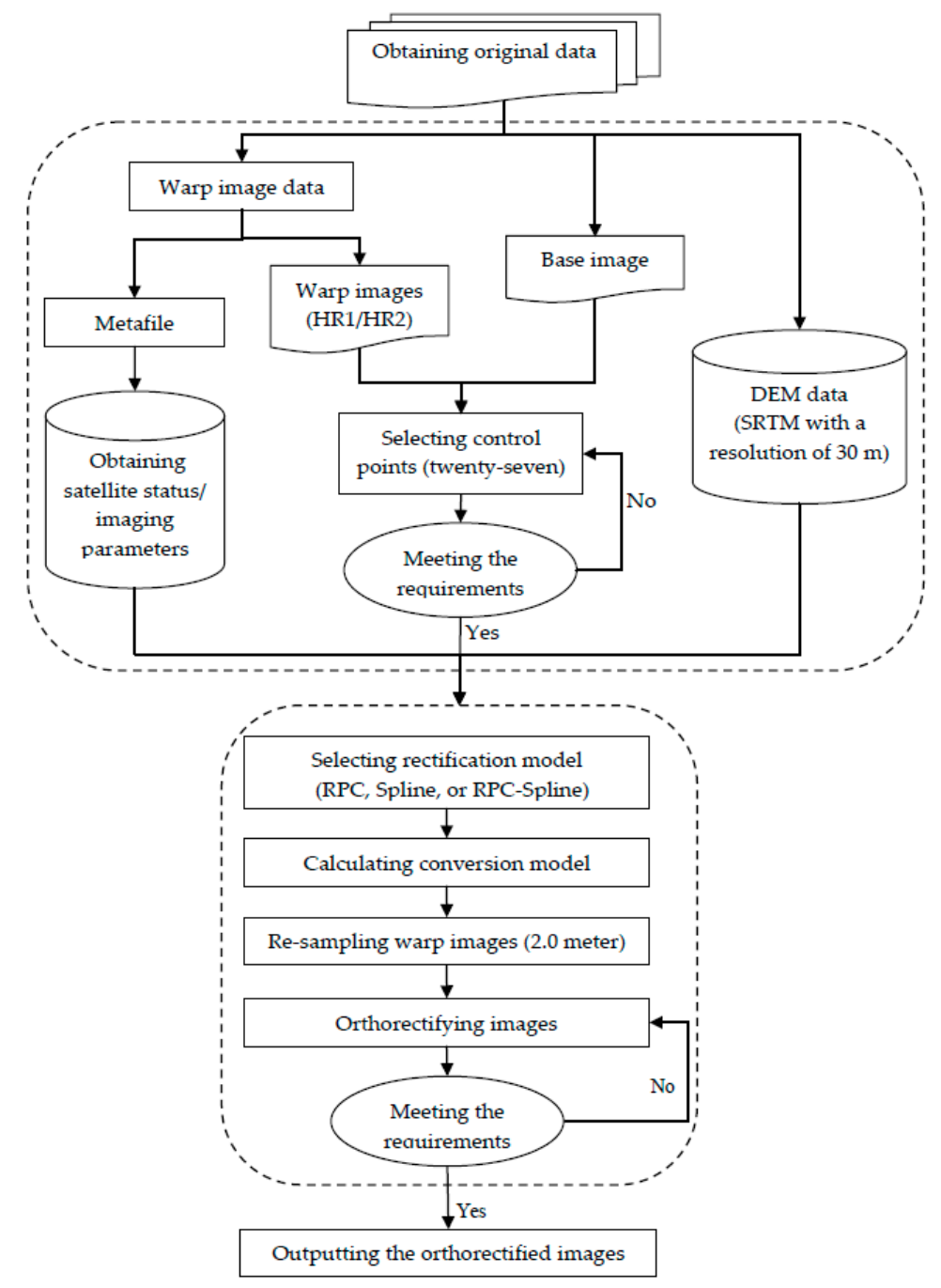

Figure 2. The procedure used for orthorectifying images in this study.

CBERS-02C HR images acquired in the study area were used to test the procedure in three operational steps: (1) executing the PCI-RPC tool; (2) executing the ArcGIS-Spline tool; and (3) executing both the PCI-RPC and ArcGIS-Spline tools. The consistent ground control points (GCPs) used for processing images with the three steps were identified and collected for comparison, analysis and verification of orthorectified images created by the three operational steps. In mountainous and hilly areas, the number of GCPs for calibration should be more than that required in plain areas [18,42]. When selecting GCPs from the both base image and warp images, the locations of GCPs should meet the following requirements:

- $\quad$ All points should be evenly distributed to represent the entire experimental area [42,43];

- Selected feature points should be clear enough to distinguish, such as road intersections, bridges corners, stadium horns, building corners, and wall angle positions, etc. [18,25];

- It is necessary to select a certain number of GCPs at the mountainside and mountaintop [18,44]; 
- The mean residual errors (i.e., RMSEs) of GCPs should be controlled in one pixel in the plains and hills, two pixels in the mountains. In this study, because the re-sampling pixel size is $2.0 \mathrm{~m}$ and the study area was featured with mountainous areas mixed with a portion of plain area, the RMSEs of GCPs should be controlled from $2.0 \mathrm{~m}$ to $4.0 \mathrm{~m}[18,45]$.

\section{Experiment and Results}

Given the mountainous areas in the study area, the same locations of the 27 GCPs were identified and selected from the base image and the warp images (or the orthorectified images created using PCI-RPC model) for all three operation steps for calibrating orthorectification models and tools. The spatial distribution of the 27 GCPs located on the base image was shown in Figure 3. Three sets of orthorectified images processed by the PCI-RPC tool, ArcGIS-Spline tool and integrating PCI-RPC and ArcGIS-Spline tools, respectively, were obtained. Their rectified accuracies were verified, analyzed and compared.

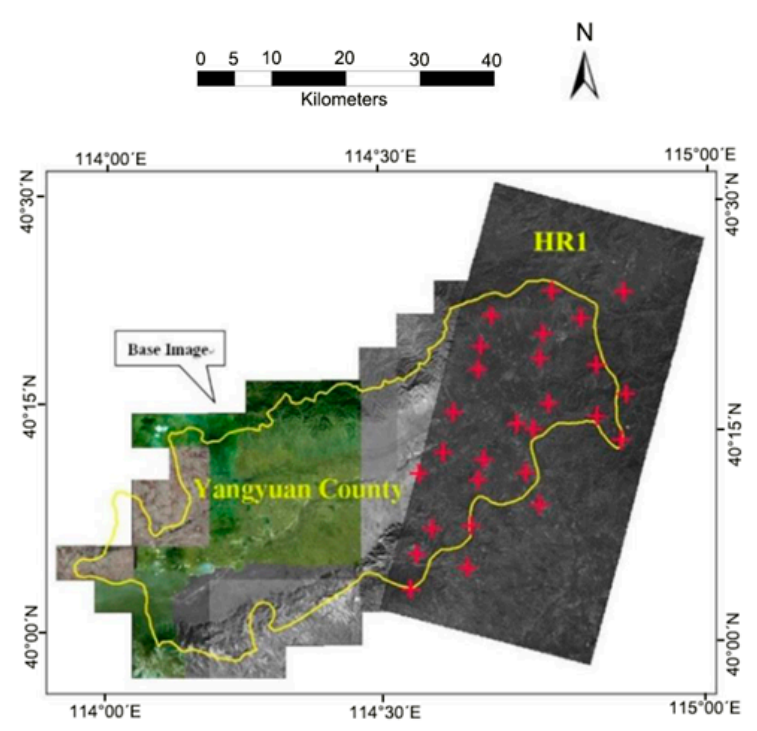

(a)

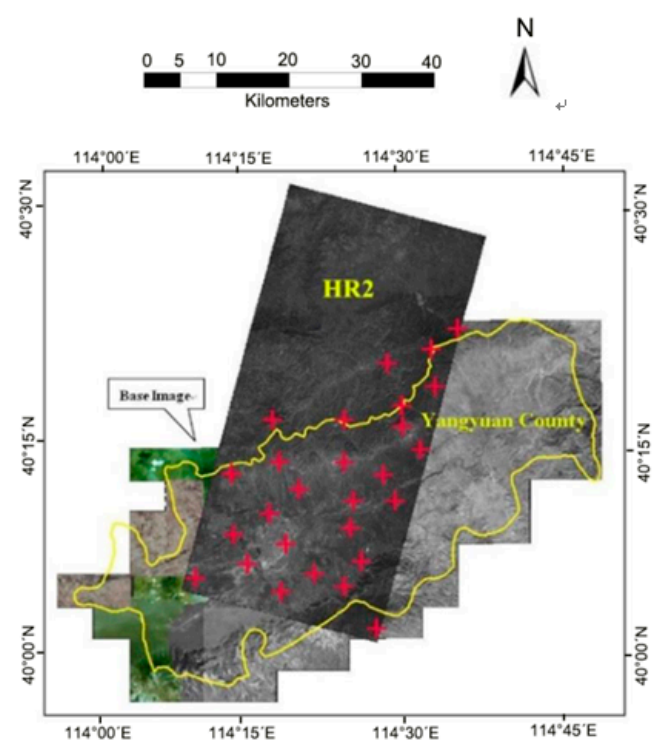

(b)

Figure 3. The spatial distribution of the rectification GCPs located on the base image: (a) the rectification GCPs were used for rectifying image HR1; (b) the rectification GCPs were used for rectifying image HR2.

\subsection{Orthorectification by PCI-RPC Tool}

At the first operation step, the satellite CBERS-02C HR images HR1 and HR2 were orthorectified by using the PCI-RPC model with the base image and DEM data. After re-sampling to $2.0 \mathrm{~m}$ pixel size by cubic convolution and calculating RMSEs [13], the orthorectified images a1 and a2 (corresponding to HR1 and HR2) were produced with the ArcGIS format of "img". Table 1 reports the residual errors of GCPs of orthorectified images a1 and a2.

Table 1. A summary of residual errors of GCPs for orthorectified images a1/a2 (unit: meter) *.

\begin{tabular}{cccccc}
\hline Model & Warp Image & Orthorectified Image & RMSE $\boldsymbol{X}$ & RMSE $\mathbf{Y}$ & RMSE \\
\hline \multirow{2}{*}{ PCI-RPC } & HR1 & a1 & 1.26 & 1.37 & 1.86 \\
& HR2 & a2 & 1.33 & 1.21 & 1.79
\end{tabular}

\footnotetext{
* "RMSE X" and "RMSE Y" represent the residual error values of GCPs in the directions of X and Y, respectively;
} "RMSE" represents the mean residual error values of GCPs. 
In Table 1, the RMSE of calibration GCPs for image a1 was $1.86 \mathrm{~m}$, and for image a2 was $1.79 \mathrm{~m}$. According to Cheng et al. [42], Wolniewicz [46], and Aguilar et al. [18], for mountainous areas, the maximum residual error should be controlled within 4 pixels, i.e., $8 \mathrm{~m}$. Thus the orthorectification accuracies of calibration GCPs had been controlled within an allowable error range.

\subsection{Geometric Correction by the ArcGIS-Spline Tool}

At the second operation step, the HR1/HR2 images were geometrically corrected based on the base image and using ArcGIS-Spline tool. In this study, since HR1/HR2 images were only given the setting of image projection parameter, the number of GCPs directly affects the geometric correction accuracy $[23,47,48]$. After calculating the RMSEs automatically (Table 2), the corresponding rectified images $b 1 / b 2$ were created. In the table, the RMSEs of GCPs were $0.4199 \mathrm{~m} / 0.2001 \mathrm{~m}$ for images $\mathrm{b} 1 / \mathrm{b} 2$, respectively, which were controlled in the allowable error range [46].

Table 2. The RMSEs of GCPs for rectified images b1/ab1, b2/ab2 (unit: meter).

\begin{tabular}{cccc}
\hline Model & Warp Image & Corrected Image & RMSE \\
\hline \multirow{2}{*}{ Spline } & HR1 & b1 & 0.4199 \\
& HR2 & b2 & 0.2001 \\
\hline \multirow{2}{*}{ RPC + Spline } & a1 & ab1 & 0.0907 \\
& a2 & ab2 & 0.0507 \\
\hline
\end{tabular}

\subsection{Integrating the PCI-RPC and ArcGIS-Spline Tools}

At the third operation step, the orthorectified images (a1/a2) created by using the PCI-RPC model were further processed by using ArcGIS-Spline tool with the same locations of calibration GCPs used for the rectification of images separately using the PCI-RPC model and ArcGIS-Spline tool, which could significantly improve the geometric corrected accuracy of the orthorectified images completed at the first operational step. The orthorectified images ab1/ab2 produced by using both PCI-RPC and ArcGIS-Spline tools were presented in Figure 4. The corresponding RMSEs of GCPs for rectified images ab1/ab2 were also listed in Table 2. In the table, it is clear that the RMSEs of GCPs were $0.0907 \mathrm{~m} / 0.0507 \mathrm{~m}$ for images ab1/ab2, respectively, which indicates that errors were controlled in the allowable error range and also much lower than those created at the second operation step.

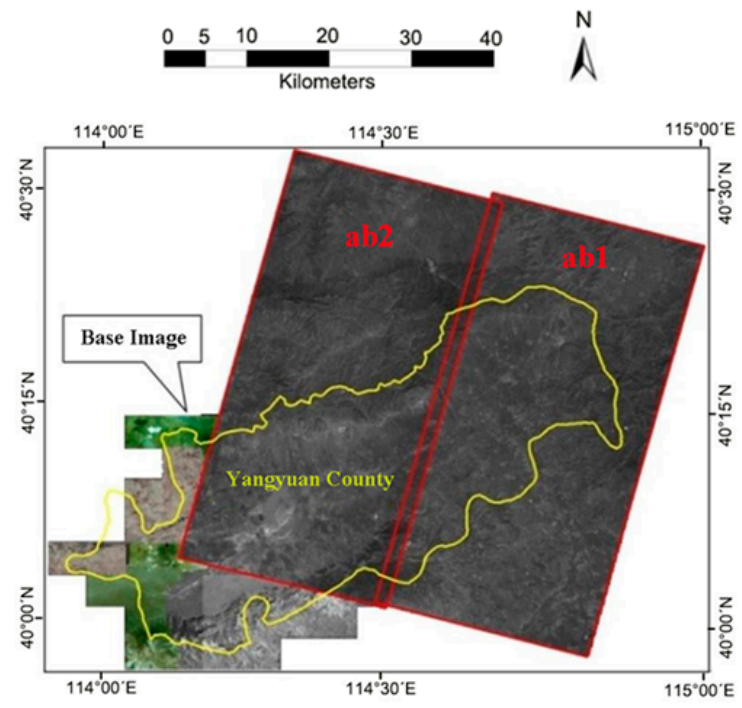

Figure 4. The improved rectification images ab1/ab2. 


\subsection{Comparison of Image Rectification Approaches}

A total of 15 validation GCPs were identified and selected from the base image and all three sets of rectified images created at the corresponding three operation steps to validate the accuracy of three sets of corrected images $\mathrm{a} 1 / \mathrm{a} 2, \mathrm{~b} 1 / \mathrm{b} 2$, and $\mathrm{ab} 1 / \mathrm{ab} 2$ (Figure 5). After automatically calculating the RMSEs of the 15 validation GCPs, their results were listed in Table 3. In the table, the RMSEs of the validation GCPs for the three sets of geometrically rectified images a1/a2, b1/b2, and ab1/ab2 were $2.94 \mathrm{~m} / 2.81 \mathrm{~m}, 4.65 \mathrm{~m} / 4.41 \mathrm{~m}$, and $1.10 \mathrm{~m} / 1.07 \mathrm{~m}$, respectively.

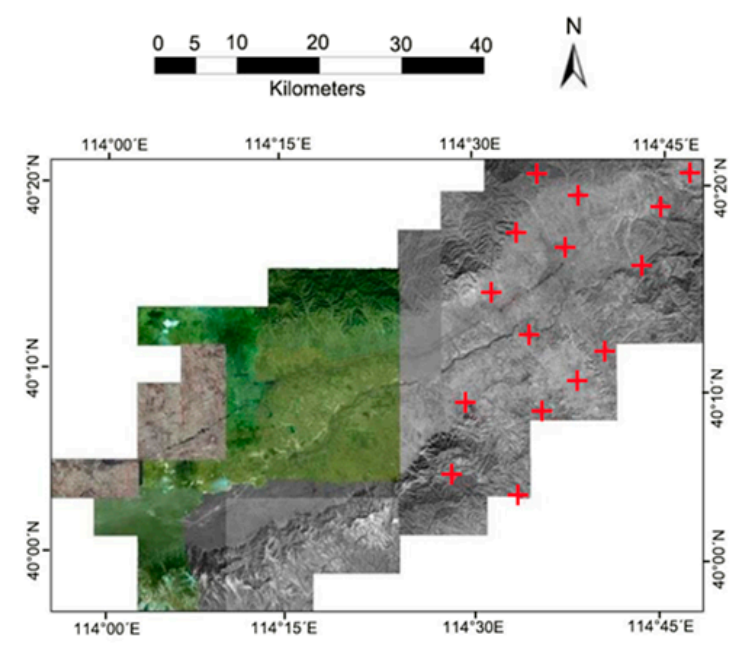

(a)

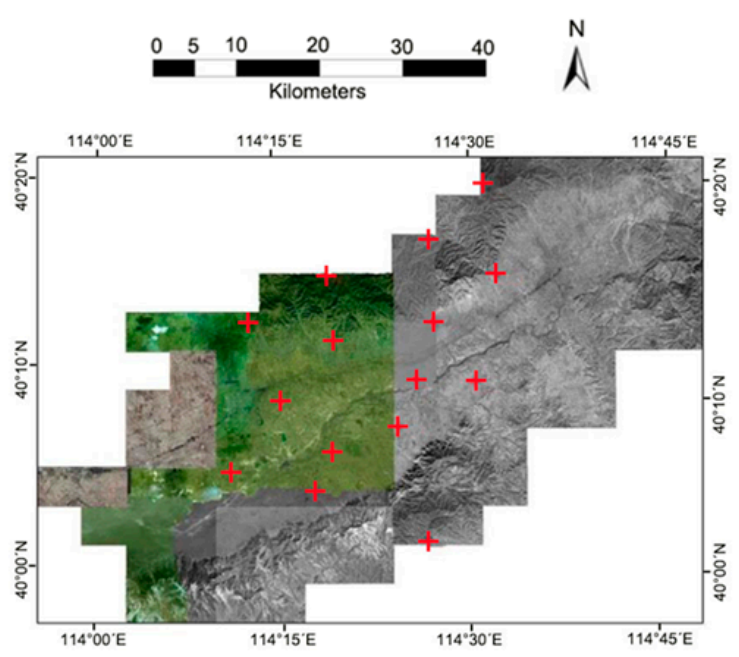

(b)

Figure 5. The spatial distribution of the validation GCPs located on the base image: (a) the validation GCPs were used for validating images a1/b1/ab1; (b) the validation GCPs were used for validating a2/b2/ab2.

Table 3. The RMSEs of 15 validation GCPs for validating rectified images a1/b1/ab1 and a2/b2/ab2 (unit: meter).

\begin{tabular}{cccccc}
\hline Modes & Warp Image & Corrected Image & RMSE $\boldsymbol{X}$ & RMSE & RMSE \\
\hline \multirow{2}{*}{ RPC } & HR1 & $\mathrm{a} 1$ & 1.78 & 2.35 & 2.94 \\
& HR2 & $\mathrm{a} 2$ & 2.06 & 1.91 & 2.81 \\
\hline \multirow{2}{*}{ Spline } & HR1 & $\mathrm{b} 1$ & 3.04 & 3.52 & 4.65 \\
& $\mathrm{HR} 2$ & $\mathrm{~b} 2$ & 3.13 & 3.10 & 4.41 \\
\hline \multirow{2}{*}{ RPC + Spline } & $\mathrm{a} 1$ & $\mathrm{ab} 1$ & 0.72 & 0.83 & 1.10 \\
& $\mathrm{a} 2$ & $\mathrm{ab} 2$ & 0.80 & 0.73 & 1.07 \\
\hline
\end{tabular}

Through the comparative analysis of verification results (Table 3) among the three sets of rectified images $\mathrm{a} 1 / \mathrm{a} 2, \mathrm{~b} 1 / \mathrm{b} 2$, and $\mathrm{ab} 1 / \mathrm{ab} 2$, the experimental results demonstrate that:

- The accuracy of validation GCPs was consistently the highest for images ab1/ab2 among the three sets of geometrically corrected images, which indicates that the orthorectification accuracy has been significantly improved by using the new image processing procedure of integrating PCI software with the RPC orthorectification model and ArcGIS-Spline tool [49];

- The accuracy of validation GCPs was consistently the lowest for images b1/b2, which means that although running the ArcGIS-Spline tool could lead to high geometrical correction accuracy for the calibration GCPs in Table 2, for the other areas in the corrected images, the geometrical correction accuracy is actually very low, as the Spline model only works well for local geometric correction around GCPs without considering the topographic relief in the study area $[20,42,43]$; 
- The accuracy of validation GCPs was secondary for images a1/a2 among the three sets of corrected images, which means that when conducting image geometric correction, incorporating DEM data (thus called orthorectification) will help improve the image geometric correction accuracy compared with the case without using DEM data in image geometric rectification.

\section{Discussion}

The test results show that the new image processing procedure which integrates PCI-RPC and ArcGIS-Spline Tools is operational and effective for improving the accuracy of image orthorectification by local geometric correction. The basic principle of the RPC model is to orthorectify images by using GCP and DEM data, and all the polynomials are the function of ground coordinates (longitude, latitude and elevation). For the image orthorectification, the projection distortion caused by the elevation difference can be rectified by the PCI-RPC with DEM data $[8,12,14]$. In the RPC model, the errors caused by an optical projection system can be expressed by a function of a rational polynomial. The errors caused by the Earth's curvature, atmospheric refraction and lens distortion can be modeled by a quadratic rational polynomial. Some other unknown error with high order components, such as camera shake, can be expressed by a cubic rational polynomial $[15,20]$.

Without sensor imaging parameters, the RPC model can not only guarantee the strict positioning accuracy with evenly distributed error, but also allow different geographic reference coordinate system. Therefore, it has great application potential in the field of high-resolution satellite imagery [26,28]. However, the accuracy of orthorectified images is still affected by the DEM accuracy. Because the RPC correction model is in the form of fraction, the denominator will change obviously when the control points used to calculate the RPC parameters are in the non-uniform distribution or excessive parameterization. Therefore, it is easy to cause the morbidity of a normal equation obtained by a modeled function, and then affect the stability of model and decrease the accuracy of image orthorectification image $[19,20,30,31]$. The calculation results obtained by orthorectifying images with the PCI-RPC model showed that the errors were obvious in both line and column directions, while the newly proposed image processing procedure integrating the PCI-RPC and ArcGIS-Spline models can correct the position errors of the RPC model. The ArcGIS-Spline model can improve the accuracy of the orthorectified image and realize the error compensation by the correction values of points with spline function.

The RPC model has 90 coefficients in total, including 10 normalized coefficients and 80 rational function coefficients. Therefore it is necessary to calculate RPC coefficients using a large number of control points $[20,30]$. In this study, experimental data of CBERS-02C HR provided with corresponding RPC coefficients, so warp images could be orthorectified by the RPC parameters with DEM data. The RPC coefficients usually have two formats, RPC text format and RPB format. Remote sensing images CBERS-02C HR provided the second format, which included 4 groups of parameters, i.e., LINE_NUM_COEF, LINE_DEN_COEF, SAMP_NUM_COEF, SAMP_DEN_COEF, corresponding to the four coefficients of Equation (2), respectively. Each group includes 20 values, corresponding to the 20 coefficients of Equation (4). Consequently, the warp images could be orthorectified by the RPC model.

Comparing the accuracy of orthorectified images obtained by using only the RPC parameters provided by satellite data providers with the base image, the orthorectified results by the RPC model showed significant displacement of control points. For example, the RMSEs of GCPs for orthorectified image HR1 in $X$ and $Y$ directions were $1.78 \mathrm{~m}$ and $2.35 \mathrm{~m}$, respectively. The RMSEs of GCPs for orthorectified image HR2 in $X$ and $Y$ directions were $2.06 \mathrm{~m}$ and $1.91 \mathrm{~m}$, respectively. A large number of studies and data analyses show that the main cause of errors in the RPC model is the re-parameterization of thephysical sensor model. The errors of both interior and exterior orientation elements may cause the errors in RPC parameters, so it is necessary and urgent to develop new methods to improve the accuracy of image orthorectification $[13,15,20]$. 
The spline function is widely used because of its simple calculation, good stability, high accuracy, certain smoothness, and the significant accuracy improvement. However, with the increase of the number of control points, the number of unknown variables is also increased. It is difficult to calculate the unknown variables with a lot of control points [42,43]. In order to solve these problems, based on the theory and method of spline function, this study adopted a cubic spline function with derivative to correct control points, which could convert the dual function into unary function to simplify the complexities of the problems, and to correct the coordinates of arbitrary points in a wide range to improve the accuracy of orthorectified images by using a local geometric correction. Compared with the base image, the results of the RPC and Spline (cubic) tools produced a dramatic accuracy improvement, and the orthorectification accuracies (RMSEs) of images HR1 and HR2 in $X$ and $Y$ directions were $0.72 \mathrm{~m} / 0.83 \mathrm{~m}, 0.80 \mathrm{~m} / 0.73 \mathrm{~m}$, respectively.

In this study, SRTM data with a $30 \mathrm{~m}$ spatial resolution were collected as DEM data, up to now, which are the best DEM data we could use in the study area. If the higher spatial resolution DEM data are available for orthorectifying images, a better result for image orthorectification may be expected. However, this does not influence the performance of the proposed procedure for improving image orthorectification.

In this study, the experimental results fully demonstrate that the new procedure has a potential to improve the accuracy of rectifying images by using PCI and ArcGIS existing software products. The new image processing procedure integrating the PCI-RPC model and ArcGIS-Spline tool has resulted in the best image orthorectification result. The improvement of image geometric rectification may be explained by the following three points:

(1) The software PCI with RPC orthorectification model considers three dimensional factors $(X, Y$, and $Z$ coordinates) and the whole image scene for an image orthorectification. Given the great topographic relief in our study area (a mountainous area), if a geometric correction model only considers correcting $X-Y$ two dimensional distortion for a warp image, it may be work well in a plain region. However, such a model for a mountainous area might be expected to work poorly due to not considering image distortion caused by the elevation variation. Thus, compared to the geometric correction result created by using ArcGIS-Spline which just considers $X-Y$ two dimensional distortion of a warp image, the PCI-RPC model outperformed the ArcGIS-Spline (Table 3).

(2) The ArcGIS-Spline tool only considers two dimensions ( $X$, and $Y$ coordinates) and local areas around GCPs (i.e., local geometric correction). The Spline function can work well in a plain area in considering image two-dimensional distortion and may result in high local geometrical correction accuracy around GCPs [46]. Consequently, the ArcGIS-Spline could create a very good geometrical correction result for the set of calibration GCPs (Table 2), but it worked very poorly for the validation GCPs (see its worst result in Table 3).

(3) The new image processing procedure that integrates PCI with the RPC orthorectification model and ArcGIS-Spline tool has a synergic advantage from the RPC model (performing a three-dimensional correction over the whole scene) and Spline tool (performing a two-dimensional correction working very well over a local area around GCPs). In this study, the orthorectified images (a1/a2) created by the PCI-RPC tool has been corrected for most three dimensional distortions at the first operation step, especially correcting the distortion caused by the elevation variation in a mountainous area. Therefore, continuously correcting the corrected images (a1/a2) with the ArcGIS-Spline tool (i.e., further correcting remained distortion at the $X-Y$ dimension) might be expected to further improve the image orthorectification accuracy. The lowest RMSEs of validation GCPs for the corrected images (ab1/ab2) in Table 3 supported this expectation.

\section{Conclusions}

In this study, we analyzed various reasons for geometric distortions, identified the differences in typical models of image geometric rectification, and discussed the definitions and rectification theories of the Rational Polynomial Coefficients (RPC) model and Spline function. Relevant disadvantages 
and difficulties were discussed for executing the RPC model and Spline function using commercial software products for orthorectifying high-resolution satellite images. A new processing procedure was proposed by integrating PCI software with the RPC model (PCI-RPC) and ArcGIS with the Spline tool (ArcGIS-Spline) to improve the accuracy of image orthorectification. The new image processing procedure was tested using two scenes of high-resolution satellite images that were acquired from a mountainous area. The experimental results demonstrated that the newly proposed procedure could significantly improve the image orthorectification accuracy by comparing with the traditional procedures such as using either the PCI-RPC model or ArcGIS-Spline function. They suggest that the new procedure would have a broad potential application, and thus it is worthy and valuable to research and develop.

With the widening application of high-resolution satellite imagery, using existing commercial image processing packages, the development of operational and efficient satellite image processing procedures such as high accurate image orthorectification will benefit those users who have a limited knowledge of remote sensing image processing.

Acknowledgments: This work is supported in part by China Scholarship Council and the National Natural Science Foundation of China under Grant No. 41471355 and 41301477. The CBERS-02C HR image data were provided by Beijing Zenith Bodhi Science and Technology Co., Ltd., Beijing, China. We also thank Yanbin Li, Beijing Zenith Bodhi Science and Technology Co., Ltd., and Baodong Ma, Northeastern University, Shenyang, China, for their valuable comments on the paper in its early version.

Author Contributions: Hongying Zhang wrote the main manuscript text and prepared the figures and tables. Ruiliang Pu contributed to the analysis and manuscript improvements and reviewing the manuscript. Xiuguo Liu supervised the research.

Conflicts of Interest: The authors declare no conflict of interest.

\section{References}

1. Jaiswal, R.K.; Saxena, R.; Mukherjee, S. Application of remote sensing technology for land use/land cover change analysis. J. Indian Soc. Remote Sens. 1999, 27, 123-128. [CrossRef]

2. Boccardo, P.; Mondino, E.B.; Tonolo, F.G.; Lingua, A. Orthorectification of high resolution satellite images. ISPRS Congr. 2004, 35, 1682-1750.

3. Kali, E.S.; Leif, G.O.; Nathan, J.H.; Patrick, L.B.; Marvin, E.B. Extending satellite remote sensing to local scales: Land and water resource monitoring using high-resolution imagery. Remote Sens. Environ. 2003, 88, $144-156$.

4. Kasper, J.; Nicholas, C.C.; Sarah, E.G.; Yulia, S. Application of high spatial resolution satellite imagery for riparian and forest ecosystem classification. Remote Sens. Environ. 2007, 110, 29-44.

5. Duccio, R. Effects of spatial and spectral resolution in estimating ecosystem $\alpha$-diversity by satellite imagery. Remote Sens. Environ. 2007, 111, 423-434.

6. Leprince, S.; Barbot, S.; Ayoub, F.; Avouac, J.P. Automatic and precise orthorectification, coregistration, and subpixel correlation of satellite images, application to ground deformation measurements. IEEE Trans. Geosci. Remote Sens. 2007, 45, 1529-1558. [CrossRef]

7. Chmiel, J.; Kay, S.; Spruyt, P. Orthorectification and geometric quality assessment of very high spatial resolution satellite imagery for Common Agricultural Policy purposes. In Proceedings of the XXth ISPRS Congress, Istanbul, Turkey, 12-23 July 2004.

8. Nichol, J.E.; Shaker, A.; Wong, M.-S. Application of high-resolution stereo satellite images to detailed landslide hazard assessment. Geomorphology 2006, 76, 68-75. [CrossRef]

9. Dowman, I.; Dare, P. Automated procedures for multisensor registration and orthorectification of satellite images. Int. Arch. Photogramm. Remote Sens. 1999, 32, 4-3.

10. Davis, C.H.; Wang, X. Urban land cover classification from high resolution multi-spectral Ikonos imagery. In Proceedings of the IEEE International Geoscience and Remote Sensing Symposium, Piscataway, NJ, USA, 24-28 June 2002.

11. Marsetic, A.; Ostir, K.; Fras, M.K. Automatic orthorectification of high-resolution optical satellite images using vector roads. IEEE Trans. Geosci. Remote Sens. 2015, 53, 6035-6047. [CrossRef] 
12. Chen, L.-C.; Teo, T.-A.; Rau, J.-Y. Optimized patch back projection in orthorectification for high resolution satellite images. Int. Arch. Photogramm. Remote Sens. 2004, 35, 586-591.

13. Fraser, C.S.; Dial, G.; Grodecki, J. Sensor orientation via RPCs. ISPRS J. Photogramm. Remote Sens. 2006, 60, 182-194. [CrossRef]

14. Grodecki, J.; Dial, G. IKONOS geometric accuracy. In Proceedings of the Joint Workshop of ISPRS Working Groups I/2, I/5 and IV/7 on High Resolution Mapping from Space, Athens, GE, USA, 29-31 October 2001.

15. Hu, Y.; Tao, C.V. Updating solutions of the rational function model using additional control information. Photogramm. Eng. Remote Sens. 2002, 68, 715-724.

16. Je, C.; Park, H.-M. Homographic p-norms: Metrics of homographic image transformation. Signal Process. Image Commun. 2015, 39, 185-201. [CrossRef]

17. DeTone, D.; Malisiewicz, T.; Rabinovich, A. Deep Image Homography Estimation. Available online: https:/ /arxiv.org/abs/1606.03798 (accessed on 8 October 2016).

18. Aguilar, M.A.; Aguera, F.; Aguilar, F.J.; Carvajal, F. Geometric accuracy assessment of the orthorectification process from very high resolution satellite imagery for Common Agricultural Policy purposes. Int. J. Remote Sens. 2008, 29, 7181-7197. [CrossRef]

19. Topan, H.; Oruc, M.; Taskanat, T.; Cam, A. Combined efficiency of RPC and DEM accuracy on georeferencing accuracy of orthoimage: Case study with pleiades panchromatic mono image. IEEE Geosci. Remote Sens. 2014, 11, 1148-1152. [CrossRef]

20. Tao, C.V.; Hu, Y. A comprehensive study of the rational function model for photogrammetric processing. Photogram. Eng. Remote Sens. 2001, 67, 1347-1357.

21. Fabio, M.A.F.; Alexander, P.T.; Konstantin, V.K.; Yi, L.; Stefan, W. Impact of orthorectification and spatial sampling on maximum NDVI composite data in mountain regions. Remote Sens. Environ. 2009, 113, 2701-2712.

22. Sowter, A. Orthorectification and interpretation of differential InSAR data over mountainous areas: A case study of the May 2008 Wenchuan Earthquake. Int. J. Remote Sens. 2010, 31, 3435-3448. [CrossRef]

23. Childs, C. Interpolating surfaces in ArcGIS spatial analyst. ArcUser 2004, 32-35.

24. Yang, J.S.; Wang, Y.Q.; August, P.V. Estimation of land surface temperature using spatial interpolation and satellite-derived surface emissivity. J. Environ. Inform. 2004, 4, 37-44. [CrossRef]

25. Chen, L.-C.; Teo, T.-A.; Wen, J.-Y.; Rau, J.-Y. Occlusion-compensated true orthorectification for high-resolution satellite images. Photogramm. Rec. 2007, 22, 39-52. [CrossRef]

26. Wu, J.; Gu, X.; Yu, T.; Meng, Q.; Chen, L.; Li, L.; Gao, H.; Wu, S. Inversion and validation of leaf area index based on CBERDS02B image data in GuangXi province of China. In Proceedings of the International Workshop on Earth Observation and Remote Sensing Applications (EORSA), Piscataway, NJ, USA, 30 June-2 July 2008.

27. Dowman, I.; Dolloff, J.T. An evaluation of rational functions for photogrammetric restitution. Int. Arch. Photogram. Remote Sens. 2000, 33, 254-266.

28. Shaker, A.A.; Eisagheer, A.A.; Eishehaby, A.R.; Mahmoud, M.S. Accuracy investigation of the orthorectification strategies for high resolution satellite images. Civ. Eng. Res. Mag. 2004, 26, 918-934.

29. Li, R.; Zhou, F.; Niu, X.; Di, K. Integration of Ikonos and QuickBird imagery for geopositioning accuracy analysis. Photogram. Eng. Remote Sens. 2007, 73, 1067-1074.

30. Aguilar, M.A.; Saldana, M.M.; Aguilar, F.J. Assessing geometric accuracy of the orthorectification process fromGeoEye-1 and WorldView-2 panchromatic images. Int. J. Appl. Earth Obs. Geo inf. 2013, 21, 427-435. [CrossRef]

31. Brink, S. Designing iterative decoding schemes with the extrinsic information transfer chart. Int. J. Electron. Commun. 2000, 54, 389-398.

32. Silva, I.D. Noise and Object Elimination from Automatic Correlation Data Using aSpline Function. Int. Arch. Photogram. Remote Sens. 1993, 29, 303-310.

33. Zhao, J.; Huang, D. Mirror extending and circular spline function for empirical mode decomposition method. J. Zhejiang Univ. Sci. A 2001, 2, 247-252. [CrossRef]

34. Spitzbart, A. A generalization of Hermite's interpolation formula. Am. Math. Mon. 1960, 67, 42-46. [CrossRef]

35. Schoenberg, I.J. Spline functions and the problem of graduation. Proc. Natl. Acad. Sci. USA 1964, 52, 947-950. [CrossRef] [PubMed] 
36. Swartz, B.K.; Varga, R.S. Error bounds for spline and L-spline interpolation. J. Approx. Theory 1972, 6, 6-49. [CrossRef]

37. Heinzl, H.; Kaider, A. Gaining more flexibility in Cox proportional hazards regression models with cubic spline functions. Comput. Methods Programs Biomed. 1997, 54, 201-208. [CrossRef]

38. Gene, D.; Howard, B.; Frank, G.; Jacek, G.; Rick, O. IKONOS satellite, imagery, and products. Remote Sens. Environ. 2003, 88, 23-36.

39. De Leeuw, A.J.; Veugen, L.M.M.; Van Stokkom, T.C. Geometric correction of remotely-sensed imagery using ground control points and orthogonal polynomials. Int. J. Remote Sens. 1988, 9, 1751-1759. [CrossRef]

40. Toutin, T. Review article: Geometric processing of remote sensing images: Models, algorithms and methods. Int. J. Remote Sens. 2004, 25, 1893-1924. [CrossRef]

41. Wang, T.; Zhang, G.; Li, D.; Tang, X.; Jiang, Y.; Pan, H.; Zhu, X. Planar block adjustment and orthorectification of ZY-3 satellite images. Photogramm. Eng. Remote Sens. 2014, 80, 559-570. [CrossRef]

42. Cheng, P.; Toutin, T. Ortho Rectification and DEM generation from high resolution satellite data. In Proceedings of the 22nd Asian Conference on Remote Sensing, Singapore, 5-9 November 2001.

43. Zhou, G.; Ron, L. Accuracy evaluation of ground points from IKONOS high-resolution satellite imagery. Photogram. Eng. Remote Sens. 2000, 66, 1103-1112.

44. Badurska, M. Orthorectification and geometric verification of high resolution terra SAR-X images. Geomat. Environ. Eng. 2011, 5, 13-25.

45. Nekrassov, V.V.; Chekalin, V.F.; Moltchachkine, N.M. Ortho/Z-space software: Highly accurate orthorectification of very high resolution satellite images. Int. Arch. Photogram. Remote Sens. Spat. Inf. Sci. 2003, 34, 1150-1153.

46. Wolniewicz, W. Assessment of geometric accuracy of VHR satellite images. Int. Arch. Photogram. Remote Sens. Spat. Inf. Sci. 2004, 34, 12-23.

47. Inglada, J.; Muron, V.; Pichard, D.; Feuvrier, T. Analysis of artifacts in subpixel remote sensing image registration. IEEE Trans. Geo Sci. Remote Sens. 2007, 45, 254-264. [CrossRef]

48. Brook, A.; Ben-Dor, E. Automatic registration of airborne and space borne images by topology map matching with SURF processor algorithm. Remote Sens. 2001, 3, 65-82. [CrossRef]

49. Chisholm, N.W.T.; Collin, R.L. Artificial GCPs in aircraft and satellite scanner imagery. Remote Sens. 1998, 9, 799-821. [CrossRef]

(C) 2016 by the authors; licensee MDPI, Basel, Switzerland. This article is an open access article distributed under the terms and conditions of the Creative Commons Attribution (CC-BY) license (http://creativecommons.org/licenses/by/4.0/). 